\title{
Radio Pulses along the Galactic Plane
}

\author{
D. J. Nice \\ Joseph Henry Laboratories and Physics Department \\ Princeton University, Princeton, NJ 08544
}

\begin{abstract}
We have surveyed $68 \mathrm{deg}^{2}$ along the Galactic Plane for single, dispersed radio pulses. Each of 3027 independent pointings was observed for $67 \mathrm{~s}$ using the Arecibo telescope at $430 \mathrm{MHz}$. Spectra were collected at intervals of $0.5 \mathrm{~ms}$ and examined for pulses with duration 0.5 to $8 \mathrm{~ms}$. Such single pulse analysis is the most sensitive method of detecting highly scattered or highly dispersed signals from pulsars with large pulse-topulse intensity variations. A total of 36 individual pulses from five previously known pulsars were detected, along with a single pulse not associated with a previously known source. Follow-up observations discovered a pulsar, PSR J1918+08, from which the pulse originated. This pulsar has period $2.130 \mathrm{~s}$ and dispersion measure $30 \mathrm{pc} \mathrm{cm}^{-3}$, and has been seen to emit single pulses with strength up to 8 times the average.
\end{abstract}

Subject headings: Pulsars: General — Pulsars: Individual (PSR J1918+08)

\section{Introduction}

Pulsars were first discovered by the examination of time series of radio total power measurements (chart recorder output) for pulses (Hewish et al. 1968). Since pulsar signals are expected to be periodic, modern pulsar searches have eschewed this single-pulse scheme in favor of searches for periodic signals, using Fourier or periodogram techniques, combined with a suitable de-dispersion algorithm. Use of a periodic search scheme typically leads to a large increase in sensitivity, as it incorporates many pulses from a single source, and the signal-to-noise ratio scales as $n^{1 / 2}$ where $n$ is the number of pulses observed.

Under certain circumstances periodic schemes fail. Very long period pulsars may be missed because there are few pulses in an observation, and pulse-to-pulse intensity variations weaken the periodic signal; in addition terrestrial radio frequency interference tends to obscure weak, long period astrophysical signals. Very short period pulsars may be missed due to broadening by finite instrumental time resolution and dispersion and scattering by the interstellar medium. Under these circumstances some individual pulses may still be detectable. Pulsars are well known to exhibit pulse-to-pulse intensity variations, and at least two pulsars exhibit "giant pulses," individual pulses 
with flux densities hundreds or thousands times greater than average. If time resolution is limited to a few milliseconds by instrumentation or by interstellar propagation effects, the periodic signal of a fast pulsar will not be detectable, yet a sufficiently strong single pulse, even if broadened to a duration of several milliseconds, will still be detectable. Indeed, the first detection of time variable radio signals from the Crab pulsar consisted of only giant pulses, because the periodic signal was faster than the instrumental time resolution (Staelin \& Reifenstein 1968).

Motivated by the possibility of detecting hitherto unknown pulsars, as well as expanding the parameter space searched for transient radio signals, we have reanalyzed a set of high sensitivity pulsar search data collected along the Galactic plane. The original analysis of these data was presented in Nice, Fruchter, \& Taylor (1995, Paper I). In that work, conventional periodic search techniques detected fourteen pulsars in the portion of the data reanalyzed here. We note that a similar single pulse study of this portion of the sky, with substantially poorer time resolution, was made by Phinney \& Taylor (1979).

\section{Observations}

Observations were made with the $305 \mathrm{~m}$ radio telescope at Arecibo, Puerto Rico, at $430 \mathrm{MHz}$ at several epochs in 1990 and 1991. Details of the observations are given in Paper I. Briefly, pointings were selected from a $9^{\prime} \times 9^{\prime}$ grid at latitudes $|b|<8^{\circ}$ and longitudes $36^{\circ}<l<70^{\circ}$. Each pointing was observed for $67 \mathrm{~s}$. A correlation spectrometer synthesized 128 spectral channels across $10 \mathrm{MHz}$ passbands in right and left circular polarization. Spectra were measured at intervals of $516.625 \mu \mathrm{s}$, for a total of 131,072 spectra per pointing. Spectra from opposite polarizations were summed, reduced to four bits, and written to tape for off-line processing. The original survey covered more than 10,000 pointings. Good quality data from 3027 pointings were reanalyzed for this work (figure 臬).

\section{Analysis}

Each pointing was independently searched for pulses with duration 0.5 to $8 \mathrm{~ms}$. To search for $0.5 \mathrm{~ms}$ pulses, time series were generated at 256 trial dispersion measures by summing spectral channels after delaying them relative to one another to compensate for dispersion. The trial dispersion measures, from 0 to $127 \mathrm{pc} \mathrm{cm}^{-3}$ at intervals of $0.5 \mathrm{pc} \mathrm{cm}^{-3}$, were equivalent to delays between the highest and lowest spectral channels of 0 to 255 sample intervals. At dispersion mea-

sures above $127 \mathrm{pc} \mathrm{cm}^{-3}$, dispersion smearing was more than $1 \mathrm{~ms}$, well above the resolution needed to distinguish $0.5 \mathrm{~ms}$ pulses. To search for pulses with higher dispersion measures, as well as longer durations, adjacent sets of 2, 4, 8, and 16 spectra were summed, and the de-dispersion algorithm repeated on each set. The time resolutions and dispersion measure ranges resulting from this procedure are listed in table 11 . 
Each de-dispersed time series was normalized and searched for points substantially higher than the mean. Comparisons of detections at adjacent times and dispersion measures were made to find the time, pulse duration, and dispersion measure that gave the highest signal-to-noise ratio for a candidate pulse.

Candidate pulses with dispersion measures indistinguishable from zero were rejected, as they arise from terrestrial radio frequency interference. Typically there were one or two such pulses per pointing. The lower limits on dispersion measure listed in table 1 for each time resolution were found to be practical minimum values for distinguishing dispersed and non-dispersed signals.

Despite the rejection of non-dispersed pulses, manual inspection of power series and spectra around many remaining candidate pulses suggested that radio frequency interference was the source of a substantial number of spurious detections. Practical a posteriori cutoffs in signal-to-noise ratio were found by such manual inspections (table [1). All signals above the quoted signal to noise ratio thresholds were of astrophysical origin $(\$ 3.2)$.

\subsection{Sensitivity}

The system temperature and telescope gain varied substantially from observation to observation due to the spillover characteristics of the telescope and to variations in sky temperature along the Galactic plane. These phenomena are discussed in Paper I. To simplify analysis of the current survey, we adopt the median system equivalent noise value from that work,

$$
N=\frac{T_{\text {sys }}}{G\left(n_{p} B t\right)^{1 / 2}}=0.156 \mathrm{Jy} n^{-1 / 2},
$$

where $n$ is the number of summed samples, and $T_{\mathrm{sys}}, G, n_{p}, B$, and $t$ are the system temperature, gain, number of polarizations, bandwidth, and integration time.

We use the term "pulse strength" to refer to the flux density integrated over the duration of the pulse, that is, energy per unit area per unit bandwidth. It is convenient to work in units of $10^{-4} \mathrm{Jys}=10^{-30} \mathrm{~J} \mathrm{~m}^{-2} \mathrm{~Hz}^{-1}$. Table 1 gives the minimum detectable pulse strength for each time resolution analyzed in the search.

\subsection{Detections}

Thirty-seven pulses were detected, nearly all at the lowest time resolution ( $8 \mathrm{~ms})$. Of these pulses, 36 originated from previously known pulsars. Table 2 lists the five sources from which these pulses originated, along with all other known pulsars within $5^{\prime}$ of a pointing center (the half-power beam radius). Catalog pulse strengths given in the table are the product of the reported $400 \mathrm{MHz}$ flux density and the pulsar period from the catalog of Taylor et al. (1995). 
The detections and non-detections of known pulsars are reasonably consistent with the detection threshold of $42 \times 10^{-4}$ Jy s for 8 ms pulses, given that scintillation by the interstellar medium can

substantially increase or decrease pulsar flux densities for a given observation. Sensitivity to some of these pulsars was degraded by long periods or high duty cycles; for example, PSR J1906+1854 has a pulse width of $64 \mathrm{~ms}$, a full 8 times longer than our lowest time resolution. In principle such long pulses would be better detected by the further reducing the time resolution of the data, but in practice distinction of dispersed pulses from non-dispersed radio frequency interference becomes increasingly difficult.

In addition to signals from known pulsars, one pulse not associated with any known source was detected. This pulse was detected with signal-to-noise ratio 25 , dispersion measure $24 \mathrm{pc} \mathrm{cm}^{-3}$, at time resolution $8 \mathrm{~ms}$, when pointing towards $\alpha(2000)=19^{\mathrm{h}} 18^{\mathrm{m}} 32^{\mathrm{s}}, \delta(2000)=08^{\circ} 38^{\prime} 32^{\prime \prime}$. Manual examination of the data found the pulse width to be $5 \mathrm{~ms}$ and the dispersion measure to be closer to $28 \mathrm{pc} \mathrm{cm}^{-3}$. No other dispersed pulses were detected in this pointing, and no statistically significant periodicities were detected. However, follow-up observations found a pulsar at this position.

\section{PSR J1918+08}

The position of the pulse was observed again on 2 July 1998, using the Arecibo telescope at $433 \mathrm{MHz}$. The Penn State Pulsar Machine recorded 128-channel spectra across a $8 \mathrm{MHz}$ passband with $80 \mu$ s time resolution over an integration time of $1200 \mathrm{~s}$. Inspection of the data revealed the presence of several strong pulses, and an underlying periodicity of $2.1 \mathrm{~s}$, indicative of a radio pulsar.

Further observations were made on 21 and 22 July 1998. Each data set was folded modulo the pulsar period. A pulse profile calculated by summing all of the data is shown in figure 2. The average flux density is roughly $0.8 \mathrm{mJy}$ (due to scintillation and crude calibration procedures this is accurate to no better than a factor of 2 ).

Standard techniques were used to calculate and analyze pulse times of arrival from these data. By splitting the data into two sub-bands and measuring the arrival time offset, the dispersion measure was determined to be $30 \pm 1 \mathrm{pc} \mathrm{cm}^{-3}$. A phase connected timing solution spanning 2-22 July 1998 yielded a pulse period of $2.1296640 \pm 0.0000004$, corrected to the solar system barycenter. The uncertainty in the period is primarily due to uncertainty in the position, which is only known to the size of the telescope beam, $\pm 5^{\prime}$.

With knowledge of the pulsar period from the follow-up data, it was possible to fold the original search data at the pulsar period. The pulsar is clearly visible, with the expected pulse shape. The original pulse is aligned with the trailing component of the pulse peak.

To discern whether PSR J1918+08 has unusual intensity fluctuations, we analyzed single pulse strengths from the search and follow-up data. Strengths were measured by comparing on-pulse power (phases 0.49 to 0.53 in figure 2) with off-pulse baseline (phases 0.00 through 0.31 and 0.71 
through 1.00.) Since scintillation is expected to modulate the observed flux on a time scale of minutes, the strength of each pulse, $U$, was normalized to the average strength of the 20 nearest pulses in the data set, $\langle U\rangle$. Only clean data with sufficiently strong average pulse signal were included. The results are summarized in figure 3. The bulk of the pulses are not individually detectable, hence the wide distribution around zero. The pulse detected in the original search data had 8 times the average pulse energy. Based on the much larger sample of pulses collected in the follow-up data, it appears that such strong pulses are rare - perhaps one in several hundred pulsesand are the high end of a smooth distribution of pulse strengths. Similar intensity distributions, with occasional pulses as much as 10 times the mean strength, are a common feature of pulsars (Hesse \& Wielebinski 1974). Thus PSR J1908+08 is a slow but apparently ordinary pulsar, and its discovery was made possible by the fortuitous emission of a rare, strong pulse during the search observation.

\section{Single pulses analysis as a tool for detecting fast pulsars}

It is intriguing that, many years after the discovery of the first millisecond pulsar, no pulsars with period shorter than $1.6 \mathrm{~ms}$ have been detected. While this may be indicative of a short-period cutoff in the pulsar population, there are also severe observational biases against such a detection. Traditional pulsar searches, using periodic analysis, inevitably loose sensitivity at short periods due to a combination of instrumental and interstellar effects. Here we consider limitations on the detectability of a $1 \mathrm{~ms}$ periodic pulsar signal in our survey data.

Sample interval. The interval at which spectra were sampled was $516 \mu \mathrm{s}$, so the Nyquist frequency of the data is about $1 \mathrm{kHz}$. Thus, a $1 \mathrm{~ms}$ pulsar would be at the edge of detectability. In principle, this restriction could be removed by sampling spectra at shorter intervals.

Dispersion smearing. The bandwidth of the individual spectral channels was $78.125 \mathrm{kHz}$. At the center frequency of $430 \mathrm{MHz}$, differential dispersion delays within a single channel broaden the signal by $(8.16 \mu \mathrm{s})(\mathrm{DM})$ where $\mathrm{DM}$, the dispersion measure, is in $\mathrm{pc} \mathrm{cm}^{-3}$. Thus for smearing to be less than $1 \mathrm{~ms}$, the pulsar must have $\mathrm{DM}<123 \mathrm{pccm}^{-3}$. Figure 1 shows the distance at which $\mathrm{DM}=123 \mathrm{pc} \mathrm{cm}^{-3}$ in the region of our search, according to the interstellar electron density model of Taylor \& Cordes (1993). The electron density is highest towards the inner portion of the Galaxy, restricting the detection of a $1 \mathrm{~ms}$ pulsar to a distance of no more than $4 \mathrm{kpc}$. The effect of dispersion smearing could, in principle, be reduced by narrowing the bandwidth of the individual spectral channels, or using coherent dedispersion techniques, in either case increasing the dispersion measure at which a $1 \mathrm{~ms}$ pulsar could be detected.

Scattering. Broadening of signals by multipath scattering provides a more stringent limit on the shortest detectable period. Figure 1 shows the distance at which the scattering time scale is $1 \mathrm{~ms}$ at $430 \mathrm{MHz}$, again using the Taylor \& Cordes (1993) model. The distance limit from scattering is also no more than $4 \mathrm{kpc}$ at the lowest Galactic longitudes surveyed. This is a firm distance limit 
on any search for periodic signals at $430 \mathrm{MHz}$, and cannot be surmounted by refinements of the data acquisition system. Thus very short period pulsars beyond this distance can only be detected through unconventional means, such as the single pulse search, or by observing at a substantially higher radio frequency (at which pulsar flux densities are typically much lower.)

Of course, the detection of a pulsar through single pulses is possible only if at least one single pulse is above the detection threshold in a given observation. Here we consider the pulse strengths of two well-studied sources, the $1.6 \mathrm{~ms}$ pulsar B1937+21 and the $33 \mathrm{~ms}$ Crab pulsar, in the context of our search.

PSR B1937+21 has a flux density of 230 mJy at $430 \mathrm{MHz}$ (Foster, Fairhead, \& Backer 1991), and a period of $1.558 \mathrm{~ms}$. Its profile exhibits both a main pulse (MP) and an interpulse (IP). Cognard et al. (1996) find that the relative strengths of the MP and IP are about 5 to 3 , and each exhibits giant pulses with the cumulative probability distribution $f=f_{0}(U /\langle U\rangle)^{\alpha}$, where $f$ is the fraction of pulses or interpulses $S$ times stronger than then mean, $\langle U\rangle, f_{0}=0.032$, and $\alpha=-1.8$. The mean pulse strength of the MP and the IP are $(5 / 8)(230 \mathrm{mJy})(1.558 \mathrm{~ms})=2.2 \times 10^{-4} \mathrm{Jys}$ and $(3 / 8)(230 \mathrm{mJy})(1.558 \mathrm{~ms})=1.3 \times 10^{-4} \mathrm{Jy} \mathrm{s}$, respectively.

A single search pointing of $67.715 \mathrm{~s}$ encompasses 43,500 rotations from this source. To estimate the strength of the strongest pulse seen in such an observation, we set $f=1 / 43500$ and solve for $U\langle U\rangle=56$. This corresponds to a MP strength of $123 \times 10^{-4} \mathrm{Jys}$, well above the single pulse detection threshold of our search. Using our highest threshold for detection of single pulses, $42 \times 10^{-4} \mathrm{Jy} \mathrm{s}$, and the distance to PSR B1937+21, $3.6 \mathrm{kpc}$, we estimate that a similar pulsar would be detectable to a distance of order $(123 / 42)^{1 / 2}(3.6)=6.2 \mathrm{kpc}$. Thus the single pulse analysis is sensitive to such pulsars at distances somewhat beyond the scattering limit of the periodic search.

PSR B0531+21, the Crab pulsar, has a flux density of $650 \mathrm{mJy}$ at $400 \mathrm{MHz}$ (Lorimer et al. 1995) and a period of $33.4 \mathrm{~ms}$. In $800 \mathrm{MHz}$ observations, Lundgren et al. 1995 found that $2.5 \%$ of the pulses are "giant" pulses, with pulse strength about 20 or more times the mean. Assuming the pulse strength ratios are similar at $430 \mathrm{MHz}$, giant pulses would have pulse strengths of at least $(20)(650 \mathrm{mJy})(33.4 \mathrm{~ms})=4300 \times 10^{-4} \mathrm{Jy} \mathrm{s}$, about 1000 times our detection threshold. Since the Crab pulsar undergoes 2000 rotations during a single search pointing, there would almost certainly be a giant pulse emitted, and it would be well above the detection threshold for any distance within the Galaxy.

We conclude that the single pulse search is a potentially powerful method of searching for fast pulsars like B0531+21 and B1937+21 in relatively distant portions of our Galaxy. The lack of detections of fast pulsars in our survey can be attributed to the small area covered by our observations, and the relatively low space density of luminous, short period pulsars.

We thank J. H. Taylor and A. S. Fruchter for collaborating in the data collection, D. Copeland for contributions towards the analysis software, and A. Wolszczan, S. B. Anderson, and B. J. Cadwell for making the Penn State Pulsar Machine available. The Arecibo Observatory is a facility of 
the National Astronomy and Ionosphere Center, operated by Cornell University under a cooperative agreement with the National Science Foundation. Pulsar research at Princeton is supported by NSF grant AST 9618357. 


\section{REFERENCES}

Cognard, I., Shrauner, J. A., Taylor, J. H., \& Thorsett, S. E. 1996, Astrophys. J., 457, L81

Foster, R. S., Fairhead, L., \& Backer, D. C. 1991, Astrophys. J., 378, 687

Hesse, K. H. \& Wielebinski, R. 1974, Astr. Astrophys., 31, 31

Hewish, A., Bell, S. J., Pilkington, J. D. H., Scott, P. F., \& Collins, R. A. 1968, Nature, 217, 709

Lorimer, D. R., Yates, J. A., Lyne, A. G., \& Gould, D. M. 1995, Mon. Not. R. astr. Soc., 273, 411

Lundgren, S. C., Cordes, J. M., Ulmer, M., Matz, S. M., Lomatch, S., Foster, R. S., \& Hankins, T. 1995, Astrophys. J., 453, 433

Nice, D. J., Fruchter, A. S., \& Taylor, J. H. 1995, Astrophys. J., 449, 156

Phinney, S. \& Taylor, J. H. 1979, Nature, 277, 177

Staelin, D. H. \& Reifenstein, III, E. C. 1968, Science, 162, 1481

Taylor, J. H. \& Cordes, J. M. 1993, Astrophys. J., 411, 674

Taylor, J. H., Manchester, R. N., Lyne, A. G., \& Camilo, F. 1995, Unpublished (available at http://pulsar.princeton.edu/ftp/pub/catalog) 


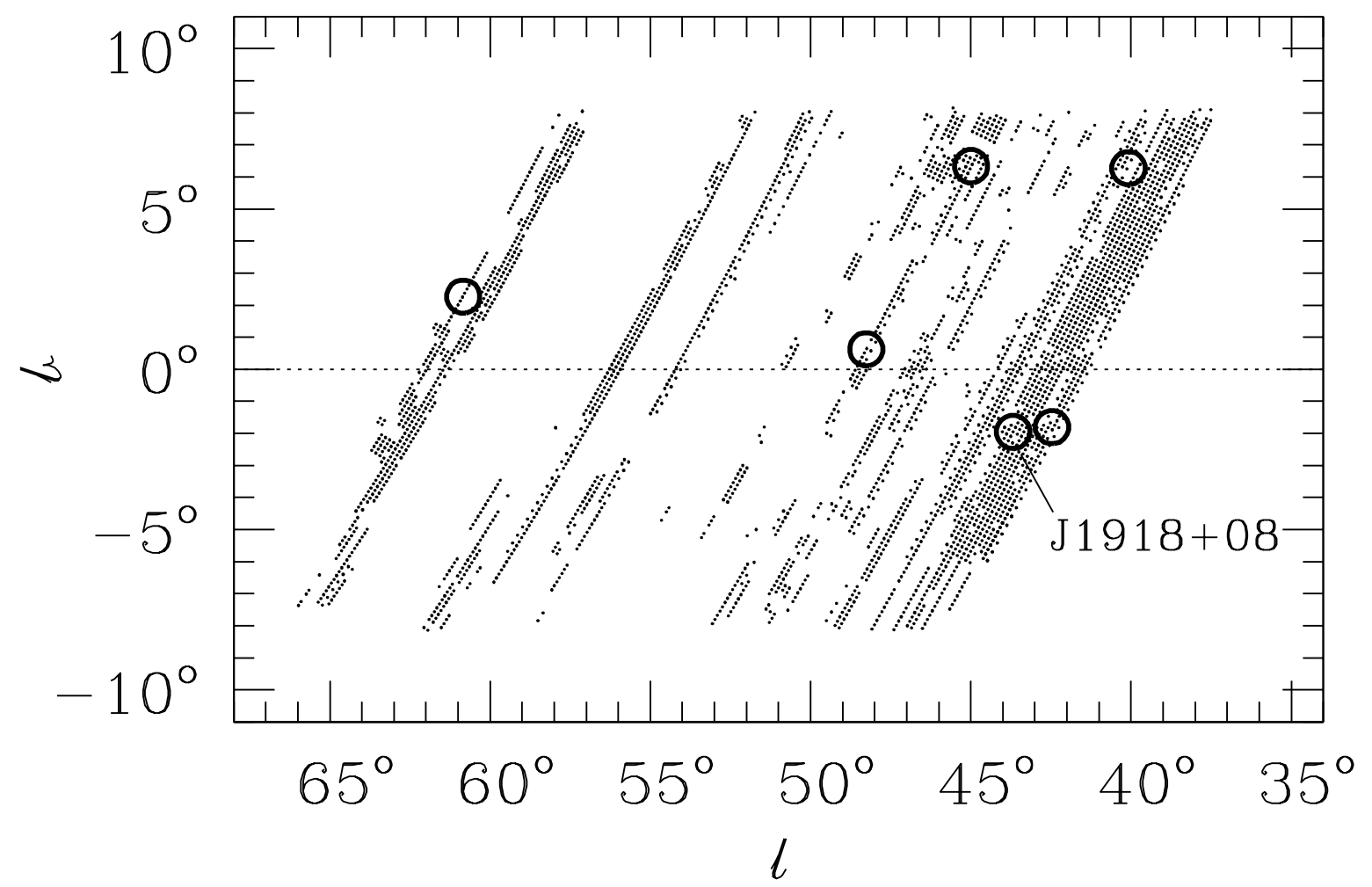

Fig. 1. - Coverage of the search in Galactic coordinates. Search pointings are indicated by small dots. Detected pulsars are indicated by large open circles. The newly discovered pulsar J1918+08 is indicated by name. 

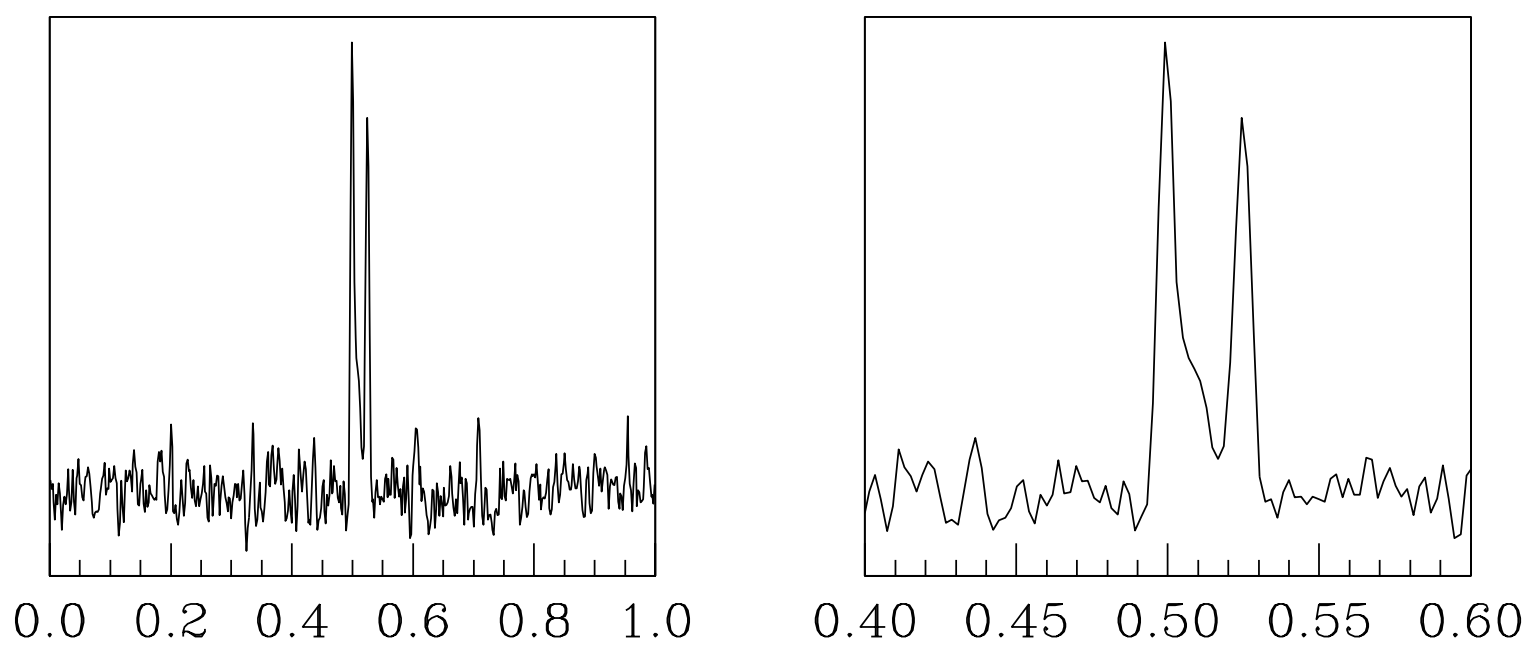

Fig. 2.- Pulse profile of PSR J1918+08 at $433 \mathrm{MHz}$. Left - full profile. Right - expanded view around pulse peak 


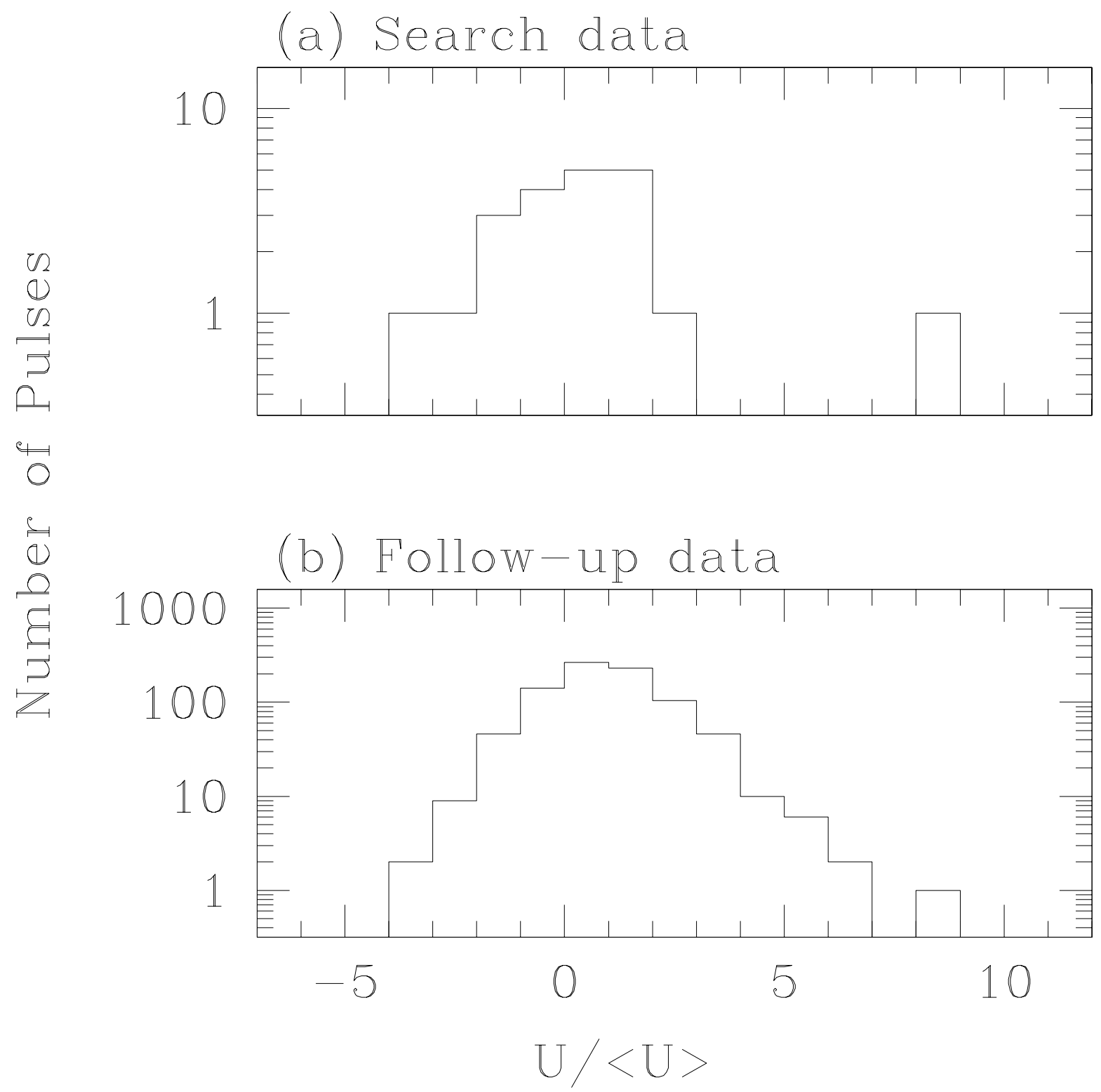

Fig. 3.- Histograms of single pulse strengths. Top - original search data. Bottom-follow-up data. 

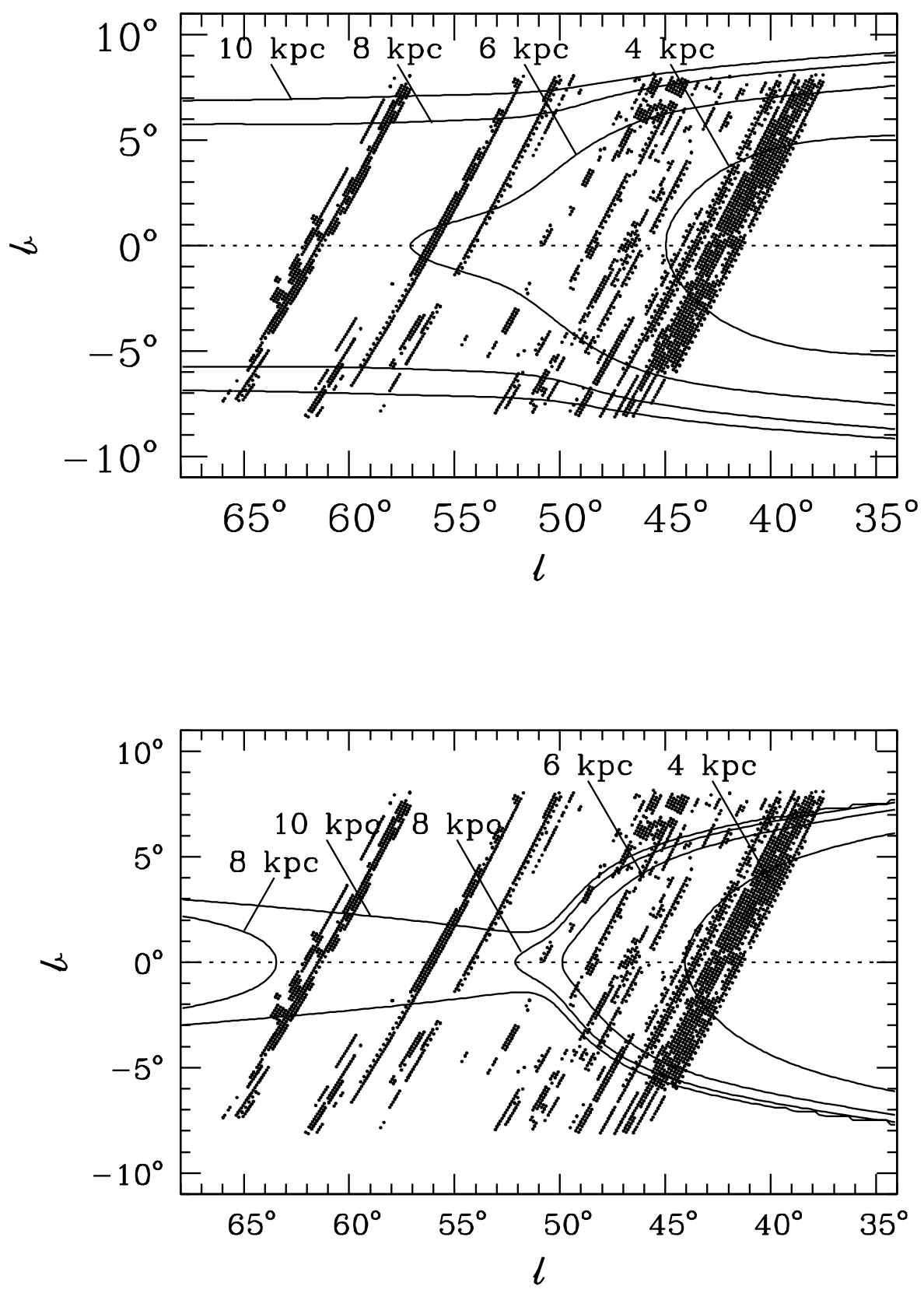

Fig. 4.- Top - Distance at which differential dispersion delay is $1 \mathrm{~ms}$. Bottom-Distance at which multipath scattering broadening is $1 \mathrm{~ms}$. 
Table 1. Search Parameters

\begin{tabular}{rccccc}
\hline \hline Samples & $\begin{array}{c}\text { Time } \\
\text { Resolution } \\
(\mathrm{ms})\end{array}$ & $(S / N)_{\text {min }}$ & $\begin{array}{c}\text { Flux Density } \\
\text { Threshold } \\
(\mathrm{Jy})\end{array}$ & $\begin{array}{c}\text { Pulse Strength } \\
\text { Threshold } \\
\left(10^{-4} \mathrm{Jys}\right)\end{array}$ & $\begin{array}{c}\text { Dispersion Measure } \\
\text { Range } \\
\left(\mathrm{pc} \mathrm{cm}^{-3}\right)\end{array}$ \\
\hline 1 & 0.5 & 9 & 1.40 & 7 & $2-127$ \\
2 & 1.1 & 9 & 0.99 & 10 & $2-255$ \\
4 & 2.1 & 9 & 0.70 & 14 & $6-511$ \\
8 & 4.1 & 11 & 0.61 & 25 & $8-1021$ \\
16 & 8.3 & 13 & 0.51 & 42 & $16-2042$ \\
\hline
\end{tabular}


Table 2. Previously known pulsars within $5^{\prime}$ of pointing positions.

\begin{tabular}{|c|c|c|c|c|}
\hline PSR & $\begin{array}{l}\text { Period } \\
\text { (s) }\end{array}$ & $\begin{array}{l}\text { Catalog Pulse Strength } \\
\qquad\left(10^{-4} \mathrm{Jy} \mathrm{s}\right)\end{array}$ & $\begin{array}{c}\text { Number of Pulses } \\
\text { Detected }\end{array}$ & Notes \\
\hline B1839+09 & 0.381 & 76 & 2 & \\
\hline $\mathrm{J} 1848+0823$ & 0.329 & 9 & & \\
\hline $\mathrm{B} 1848+13$ & 0.346 & 21 & 6 & \\
\hline $\mathrm{J} 1902+9723$ & 0.488 & 3 & & \\
\hline B1906+09 & 0.830 & 42 & & 1 \\
\hline $\mathrm{J} 1906+1854$ & 1.019 & 47 & & \\
\hline B1911+09 & 1.242 & 37 & & 1 \\
\hline $\mathrm{B} 1913+10$ & 0.404 & 24 & & \\
\hline $\mathrm{J} 1915+0738$ & 1.543 & 29 & 2 & \\
\hline B1915+13 & 0.195 & 84 & 24 & \\
\hline $\mathrm{J} 1916+07$ & 0.542 & 22 & & 1 \\
\hline $\mathrm{J} 1918+0734$ & 0.212 & 7 & & \\
\hline $\mathrm{B} 1922+20$ & 0.238 & 10 & & 1 \\
\hline B1925+18 & 0.483 & 14 & & \\
\hline $\mathrm{B} 1929+20$ & 0.286 & 49 & & \\
\hline $\mathrm{B} 1935+25$ & 0.381 & 13 & 2 & \\
\hline $\mathrm{J} 1941+1026$ & 0.905 & 16 & & \\
\hline $\mathrm{J} 2008+2513$ & 0.589 & 16 & & \\
\hline $\mathrm{J} 2019+2425$ & 0.004 & 1 & & \\
\hline
\end{tabular}

Note. - 1. Pulsar not detected in periodic signal search of Paper I. 